\title{
Effects of Fruit Bagging on Anthocyanin Accumulation and Related Gene Expression in Peach
}

\author{
Yingtao Ma and Mengmeng Zhao \\ Life Science College, Luoyang Normal University, Luoyang, Henan 471934, China
}

\begin{abstract}
Hongxia Wu
South Subtropical Crops Research Institute, Chinese Academy of Tropical Agricultural Sciences, Zhanjiang, Guangdong 524091, China
\end{abstract}

Congying Yuan, Huiyun Li, and Yanzhao Zhang

Life Science College, Luoyang Normal University, Luoyang, Henan 471934, China

\begin{abstract}
AdDITIONAL INDEX wORDs. light, paper bags, skin coloration, transcript factor
Abstract. Fruit bagging is a popular agricultural practice that has been widely used to physically protect fruit. However, the application of fruit bags usually has various effects on fruit quality. In this study, three kinds of paper bags with different colors and transmittance were applied to investigate their effects on the skin coloration and related gene expression of peach (Prunus persica). Our findings showed that bagging treatment inhibited anthocyanin accumulation and the expression of related structural and regulatory genes in the peach pericarp. To a certain extent, the inhibitory effects were negatively correlated with the light transmittance of these paper bags. The expression of $M Y B 10.1$ was also suppressed by fruit bagging and was highly consistent with anthocyanin content in peach pericarps, which indicated that MYB10.1 might have a critical role in the light-mediated regulation of anthocyanin production in peach pericarps. These findings further enrich our theoretical knowledge of the regulation of anthocyanin synthesis in peach fruit and provide a theoretical basis for common horticultural practices.
\end{abstract}

As a widely grown fruit, peach (Prunus persica) is highly prized for its flavor and nutritional value. Consumer demand for high-quality peaches with no pesticide residue is increasing, but numerous diseases and insect pests make peaches one of the most heavily sprayed crops, and birds can cause serious losses when peaches are not protected. Fruit bagging is a good agricultural practice that can physically exclude pests, prevent disease development, and consequently help reduce the reliance on pesticides that have been widely used for fruit cultivation (Han et al., 2015; Sharma et al., 2014). Fruit bagging can also effectively protect fruit against physical damage from pests and birds. However, it is believed that bagging until fruit harvest is not good for color development in fruit skin. Previous studies have indicated that bagging with a light-impermeable bag significantly lowers anthocyanin concentrations in apple (Malus domestica) peels (Feng et al., 2014). The visual appearance and anthocyanin concentration of european pear (Pyrus communis) were also affected by bagging treatments (Huang et al., 2009). For peaches, it has also been demonstrated that bagging with orange bags

Received for publication 13 Nov. 2020. Accepted for publication 5 Feb. 2021.

Published online 19 May 2021.

This research was supported by the Natural Science Foundation of China (31700604), Study Abroad Project sponsored by China Scholarship Council (201808410220), Key Scientific Research Project of Henan Higher Education (16A210011), and Key Technology Project of Henan Province (192102110035).

Y.M. is the corresponding author. E-mail: mayingtao1008@hotmail.com.

This is an open access article distributed under the CC BY-NC-ND license (https://creativecommons.org/licenses/by-nc-nd/4.0/). inhibits anthocyanin accumulation in the peel (Jia et al., 2005; Liu et al., 2015).

As the main pigment in fruit, anthocyanin enriches the color of the peel and increases the attractiveness of the fruit to consumers (Jimenez-Garcia et al., 2013). Color is an important determinant of quality and ripeness (Allan et al., 2008). A variety of biological functions of anthocyanins have been demonstrated in plants, including protection against light stress, defense, the attraction of pollinators, and seed dispersal (Holton and Cornish, 1995). Findings derived from animal models, cell-line studies, and human clinical trials showed that anthocyanins comprise a class of substances beneficial to human health, including anticarcinogenic activity and anti-inflammatory, anti-aging, obesity control, cardiovascular disease prevention, and diabetes alleviation (He and Giusti, 2010; Sun et al., 2012; Zheng et al., 2020).

Anthocyanin synthesis is a metabolic pathway catalyzed by multiple enzymes, including chalcone synthase (CHS), phenylalanine ammonialyase (PAL), dihydroflavonol 4-reductase (DFR), chalcone isomerase (CHI), anthocyanidin synthase (ANS), flavonoid 3-hydroxylase (F3H), and UDP-glucose: flavonoid-3-Oglucosyltransferase (UFGT) (Jaakola, 2013; Kobayashi et al., 2001; Montefiori et al., 2011; Tanaka et al., 2008; Winkel-Shirley, 2001). Environmental factors such as low temperature and light can enhance anthocyanin accumulation in fruit (Guo et al., 2018; Liu et al., 2019a; Lo Piero et al., 2005; Zhou et al., 2013). Anthocyanin synthesis in apple peels is regulated by DFR activity under low sunlight intensity, but it is dependent on UFGT activity after exposure to high sunlight (Chen et al., 2019). UFGT is also the key enzyme regulating anthocyanin accumulation in kiwifruit [Actinidia chinensis (Liu et al., 2018)]. It has been demonstrated that the expressions of $C H S, U F G T$, and $F 3 H$ 
genes are positively correlated with the anthocyanin content of different parts of peach fruit (Rahim et al., 2014). Cao et al. (2018) suggested that F3H and DFR are rate-limiting enzymes within the anthocyanin biosynthesis process by analyzing the flesh transcriptomes of 30 peach cultivars. Liu et al. (2019b) reported that the expression patterns of $C H S$ and $D F R$ genes are consistent with changes in the anthocyanin content of ' $\mathrm{LuYou}$ Tao1' peach skin during fruit development. Tsuda et al. (2004) suggested that CHS and DFR genes are the key enzymes in the synthesis of anthocyanins in the skins of the red peach 'Akatsuki'. Another recent study of 'JinXiu' peaches also showed that DFR, UFGT, and CHS have important roles in anthocyanin accumulation in skins (Ye et al., 2019). The most critical genes appear to vary among fruit species, cultivars, and different fruit parts, and these biosynthetic genes respond differently to environmental changes. Most importantly, F3H, DFR, UFGT, and CHS were the most reported key enzymes in anthocyanin accumulation in peach. The synthetic regulation of anthocyanin appears to be primarily at the transcriptional level with transcription factor complex of basic helix-loop-helix (bHLH), R2R3-MYB, and WD40 repeat proteins (Gonzalez et al., 2008; Laitinen et al., 2008; Li et al., 2020; Peng et al., 2019; Petroni and Tonelli, 2011). A previous report stated that the WD repeat protein is constitutively expressed in plants (Glover and Martin, 2012). R2R3-MYB transcription factors have been reported to be the major determinants of the anthocyanin level by regulating key anthocyanin biosynthetic genes (Allan et al., 2008; Peng et al., 2019; Ravaglia et al., 2013; Tuan et al., 2015).

In peaches, the predominant component that contributes to the red pigmentation is cyanidin-3-glucoside [C3G (Cheng et al., 2014; Hsia et al., 1965; Reig et al., 2013)]. Fruit bagging has been reported to affect anthocyanin accumulation, but the underlying mechanism remains unclear, and it remains to be explored how different bagging treatments affect the expression patterns of biosynthetic and regulatory genes. In this study, three kinds of paper bags with different colors and transparency were used to investigate the effects of bagging on $\mathrm{C} 3 \mathrm{G}$ accumulation and the expression levels of the mostly reported major biosynthetic genes, $F 3 H, D F R, U F G T$, and $C H S$, and regulatory genes in peach pericarps.

\section{Materials and Methods}

Plant materials and bagging treatments. Extremely early-maturing peach cultivar Zaochunhong was used in this study. The 7-year-old trees were cultivated in an orchard at the Luoyang Academy of Agriculture and Forestry Science (Henan, China). As described in a previous study (Ma et al., 2018),
Zaochunhong is an early ripening peach cultivar with white firm-melting flesh, sweet flavor, and strong resistance that is derived from cross-breeding Sunago Wase and Yuhualu. A completely randomized design was performed during this experiment. Before the pit-hardening stage (38 d after anthesis), 180 fruit with uniform size and no obvious defects selected from 15 peach trees were randomly divided into four equal parts, one of which grown under normal conditions was used as a control and the other three parts were covered on the trees with commercial $17.0-\times 21.0-\mathrm{cm}$ waterproof paper bags (Laiyang Yintong Papermaking Co., Laiyang, China), including a white single-layer paper bag (W-P; $\approx 50 \%$ transmission of sunlight), a yellowwhite double-layer paper bag (YW-P; white waxy plastic inner layer and yellow paper outer layer with $\approx 18 \%$ transmission of sunlight), and a khaki-black double-layer paper bag [KB-P (black waxy plastic inner layer and khaki paper outer layer with $\approx 0 \%$ transmission of sunlight)] (Fig. 1). These selected fruits are distributed on the south or west side of the tree and vertically distributed in the upper middle position within 1.5 to $3.0 \mathrm{~m}$ of the canopy; the average relative light intensity is $\approx 49 \%$. Fruit were harvested at commercial maturity (68 d after anthesis) based on unbagged fruit. The bags on the bagged fruit were not removed until the quality analysis.

Tissue COLLECTION AND DETERMinATION OF ANTHOCYANIN CONTENT. The total pericarps of each fruit, including the outer epidermis and a few layers of hypodermal cells, were excised and collected on the day of harvest, frozen immediately in liquid nitrogen, and stored at $-80^{\circ} \mathrm{C}$ for subsequent experiments. Anthocyanin was extracted and determined as follows: frozen pericarps were ground with a pestle in a mortar using liquid nitrogen; then, $1 \mathrm{~g}$ of powdered pericarps was first incubated in $40 \mathrm{~mL}$ of extraction buffer (methanol with $0.1 \%$ hydrochloric acid) at $4{ }^{\circ} \mathrm{C}$ for $12 \mathrm{~h}$; the mixed solution was centrifuged at $4472 g_{n}$ for $5 \mathrm{~min}$; and the supernatant was collected after filtering with $0.22-\mu \mathrm{m}$ membrane filters. Quantitative detection of anthocyanin was performed by high-performance liquid chromatography (1100; Agilent Technology, Santa Clara, CA) as described previously (Fan et al., 2012). The anthocyanin content in the samples were calculated three times by a linear regression equation using pure $\mathrm{C} 3 \mathrm{G}$ as the standard. For each sample, 12 peach fruit collected from five trees were selected and analyzed as a biological replicate; for every sample, three biological replicates were analyzed.

Quantitative REAL-TIMe POlymerase CHAin REACTION ANALYSIS. Total RNA was isolated from peach peels using a modified cetyltrimethylammonium bromide method as described previously (Liu et al., 2015). The integrity and purity analyses of the purified RNA were performed with a spectrophotometer (NanoDrop 2000; Thermo Fisher Scientific, Waltham, MA). First-strand cDNA was synthesized using the RevertAid First Strand cDNA Synthesis Kit (K1621; Thermo Fisher Scientific). Quantitative real-time polymerase chain reaction (qRT-PCR) was performed with Power SYBR Green PCR Master Mix (4367659; Applied Biosystems, Foster City, CA) using a PCR instrument (CFX96; Bio-Rad, Hercules, CA) using the following protocol: $95^{\circ} \mathrm{C}$ for $3 \mathrm{~min}$; 40 cycles
Fig. 1. Different paper bags used during this study to wrap the peach fruit on the tree. Control $=$ standard conditions with no bagging treatment; W-P = white single-layer paper bag; YW-P = yellow-white doublelayer paper bag; KB-P = khaki-black double-layer paper bag.

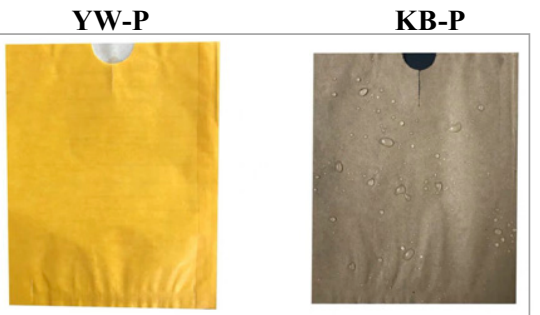


of $95^{\circ} \mathrm{C}$ for $10 \mathrm{~s}, 55^{\circ} \mathrm{C}$ for $20 \mathrm{~s}$, and $72^{\circ} \mathrm{C}$ for $5 \mathrm{~s}$; and $75^{\circ} \mathrm{C}$ for $10 \mathrm{~s}$, followed by a continuous increase from 65 to $95^{\circ} \mathrm{C}$ with a ramp rate of $0.5^{\circ} \mathrm{C} \cdot \mathrm{s}^{-1}$ for dissociation curve analysis. Each reaction was performed in a total volume of $20 \mu \mathrm{L}$ containing $2 \mu \mathrm{L}$ of diluted cDNA, $1 \mu \mathrm{L}\left(10 \mu \mathrm{mol} \cdot \mathrm{L}^{-1}\right)$ of each primer and $10 \mu \mathrm{L}$ of $2 \times$ SYBR Green PCR Master Mix. The peach gene Actin (Genome Database for Rosaceae accession no. ppa007214m) was chosen as an internal control reference gene for normalization, and the relative expression levels of mRNAs were measured using the $2^{-\Delta \Delta \mathrm{Ct}}$ method as described previously (Livak and Schmittgen, 2001). Primer pairs used for the qRT-PCR analysis are listed in Supplemental Table 1.

Statistical analysis. All data regarding the anthocyanin concentration and expression of the anthocyanin biosynthetic genes were calculated using Microsoft Excel 2010 (Microsoft Corp., Redmond, WA) and presented as the mean \pm SE. Statistical analyses were performed using Student's $t$ test and one-way analysis of variance using SPSS (version 25.0; IBM Corp., Armonk, NY). Significance is indicated by different letters.

\section{Results}

EFFECTS OF BAGGING TREATMENTS ON THE VISUAL QUALITY OF 'ZAOChUnhONG' PEACHes. The skin color of commercially mature peaches varied significantly with the bags applied, with the deepest red color developed in nonbagged control fruit (Fig. 2). The three different paper bags inhibited the color development of peach pericarps. As the light transmittance of the fruit bags decreased, the accumulation of redness in the pericarps gradually decreased. Peaches bagged with the W-P bags, which had the highest light transmission among the three types of paper bags used, showed a lighter and striped red color compared with nonbagged controls, with the red area much larger than that on fruit bagged with YW-P and KB-P bags. The red area of the pericarps of fruit bagged with YW-P bags was slightly larger than that on those bagged with KB-P bags; however, fruit bagged with two types of bags had very little accumulated redness (Fig. 2).

Anthocyanin CONTENT OF 'Zaochunhong' Peaches. The anthocyanin content of peach peels was well-correlated with visual appearance. The highest anthocyanin content was present in nonbagged peach peels and was significantly higher than that in bagged peaches (Fig. 3). The anthocyanin accumulation in peaches bagged with W-P bags decreased, whereas the pericarps of fruit wrapped with KB-P trol peach pericarps. (Fig. 4A). bags (Fig. 1).

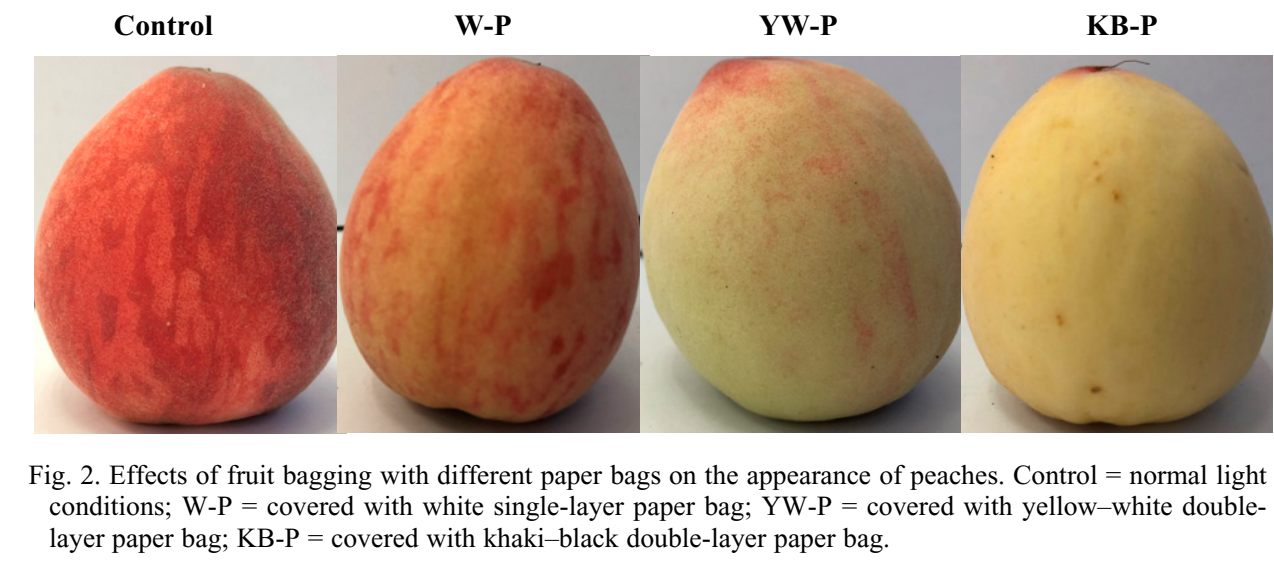

Fig. 2. Effects of fruit bagging with different paper bags on the appearance of peaches. Control = normal light conditions; W-P = covered with white single-layer paper bag; YW-P = covered with yellow-white doublelayer paper bag; KB-P = covered with khaki-black double-layer paper bag.

\section{KB-P}

and YW-P bags accumulated very little anthocyanin; no significant differences were observed between them.

EXPRESSION OF GENES RELATED TO ANTHOCYANIN BIOSYNTHESIS. Bagging treatments significantly influenced the appearance and anthocyanin content of peach peels (Figs. 2 and 3). To further understand the differences in anthocyanin accumulation, the gene expression profiles of related biosynthetic pathway genes, including $C H S, F 3 H, D F R$, and $U F G T$, and the three transcription factor-encoding genes, including bHLH3, MYB10.1, and $M Y B 10.2$, were determined for bagged peach pericarps and con-

The expressions of these four structural genes were significantly repressed by bagging (Fig. 4A). Among the selected genes, the inhibitory effects of different bags varied, but the same trend was found. Bagging treatments inhibited their expressions in peach pericarps, and the degree of inhibition was inversely related with the transmittance of the paper bag used. All four genes had the highest expression levels in the nonbagged peach pericarps, whereas the expressions in the KB-P and YW-P were the lowest. However, no significant differences were observed between the KB-P and the YW-P bagged pericarps

As shown in Fig. 4B, the expression of the transcription factor-encoding gene MYB10.2 was very low, and no consistent trend related to anthocyanin content was observed between the nonbagged and bagged pericarps, regardless of the type of bag used. The gene $b H L H 3$ was expressed at a relatively high level, but no significant influence from the bagging treatment on its expression was observed. The expression of MYB10.1 was significantly higher in the pericarps of fruit bagged with W-P than in the pericarps covered with the other two bags; however, it was much lower than that in the control and was highly correlated with the redness of the skin and the anthocyanin levels.

\section{Discussion}

The influence of fruit bagging with different paper bags on anthocyanin accumulation in 'Zaochunhong' peach pericarps was investigated during our study. To clarify the underlying mechanism, the expression profiles of the biosynthetic and regulatory genes involved in the anthocyanin pathway were determined. The results indicated that fruit bagging inhibited the synthesis of anthocyanin in the peach pericarp, and that the inhibitory effect was related to the light transmittance of the paper

Anthocyanin biosynthesis is a complicated pathway influenced by environmental factors and agricultural practices that could be induced by various stresses, such as high light exposure, low temperatures, nutrient deficiency, or pathogen attack (Dixon and Paiva, 1995). Light is one of the most important environmental factors affecting the synthesis of anthocyanins (Zoratti et al., 2014). High light exposure has been demonstrated to induce anthocyanin pigmentation in various fruits, such as pears (Bai et al., 2019), apples ( $\mathrm{Li}$ et al., 2012), and litchis [Litchi chinensis 


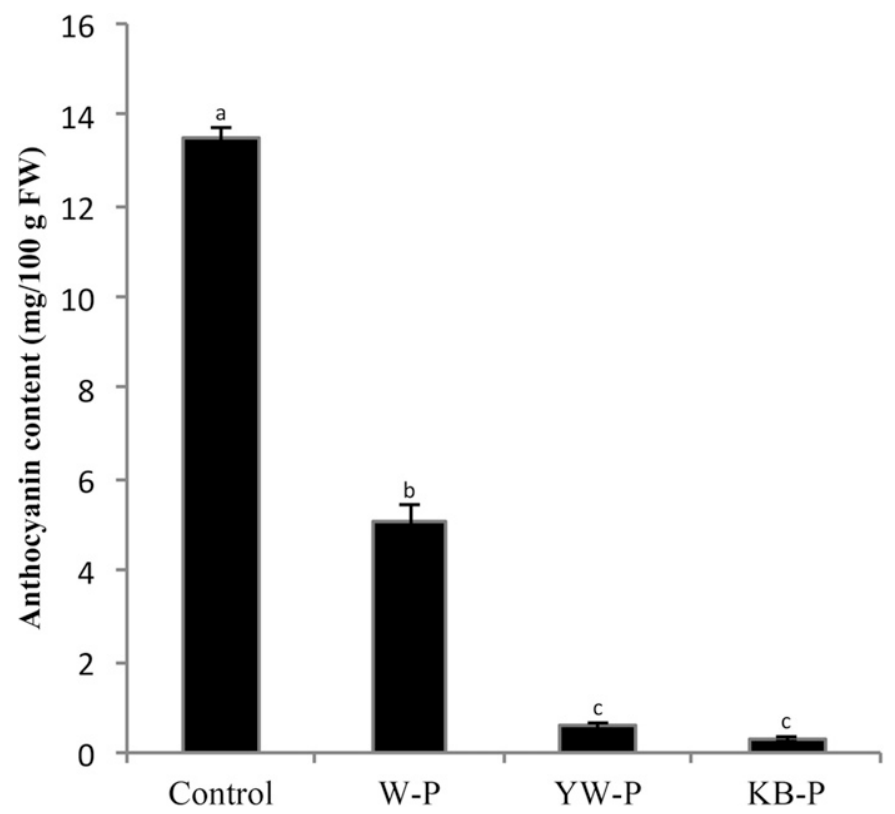

Fig. 3. Effects of fruit bagging with different bags on the cyanidin-3-glucoside $(\mathrm{C} 3 \mathrm{G})$ accumulation in peach pericarps. Control $=$ standard conditions with no bagging treatment; W-P = white single-layer paper bag; YW-P = yellow-white double-layer paper bag; KB-P = khaki-black double-layer paper bag. Data are means \pm SD of three independent biological replicates. Bars represent SE. The letters represent the nonsignificant ranges according to the least significant difference (LSD) test $(P \leq 0.01)$.

(Zhang et al., 2016)]. Reducing light irradiation inhibits anthocyanin synthesis, especially in the epidermal tissues, and this may be one reason why the pericarps of bagged fruit usually contain less anthocyanin. Fruit bagging is a popular agricultural practice that is widely used for many fruit species to protect fruit from all kinds of pests and bird damage. However, bagging provides a microenvironment with low light transmittance, which might affect anthocyanin accumulation in fruit pericarps (Feng et al., 2014; Huang et al., 2009). In peaches, the anthocyanin content in the peel decreases as the exposure to sunlight of the bagged fruit decreases (Jia et al., 2005), and the bagging of peaches with opaque paper until harvest leads to little or nearly no anthocyanins in peach skins (Ma et al., 2018). In this study, three kinds of paper bags with different colors and light transmission were used to investigate the effects of different bagging treatments on peach appearance. Compared with the W-P bags, the KB-P bags with the lowest light transmittance showed the strongest inhibitory effect on anthocyanin production in peach skins (Figs. 2 and 3). However, no significant differences in pericarp color and anthocyanin content were found between the peaches bagged with the Y-W bags and the KB-P bags, respectively. We infer that anthocyanins protect peaches from high light exposure; as such, damage to bagged fruit is reduced and the anthocyanin accumulation is reduced accordingly. However, when the light intensity is reduced to a certain level, the change in light no longer affects the anthocyanin accumulation.

The light-mediated color changes were associated with the altered expression levels of certain light-responsive anthocyanin synthesis genes. In our study, it was shown that bagging treatment, especially bagging with opaque paper bags, downregulated the expression of all the selected biosynthetic genes, including the F3H, CHS, UFGT, and DFR, the expressions of which were significantly downregulated in bagged fruit compared with the control fruit. However, fruit bagged with the KB-P and YW-P bags had the lowest transcript levels, which were highly positively correlated with anthocyanin levels in peach skin (Fig. 4A). These findings might suggest that all selected structural genes are sensitive to light change. Further studies are needed to clarify which is the critical biosynthetic gene responding to bagging treatment and resulting in low anthocyanin levels in the skin of bagged peach.

The varying expressions of the biosynthetic genes can be associated with the regulation of upstream regulators. It has been well-documented that anthocyanin synthesis is regulated by a regulatory complex consisting of bHLH transcription factors, R2R3 MYB transcription factors, and a WD repeat protein. During our research, we determined the gene expressions of the most correlated factors involved in anthocyanin biosynthesis in peaches (MYB10.1, $M Y B 10.2$, and $b H L H 3)$. The expression pattern of $b H L H 3$ in peaches with different bagging treatments was not consistent with the anthocyanin level of these peaches, with bagged peaches having a higher expression than nonbagged control peaches and no significant difference found among the differently bagged peaches. Regarding the MYB transcription factors, the transcript level of litchi R2R3-MYB LcMYB1 was positively correlated with the anthocyanin content in litchi pericarps and can be regulated by light (Lai et al., 2014); this has also been reported for apples (Takos et al., 2006) and apricots [Prunus armeniaca (Xi et al., 2019)]. During this study, the expression of MYB10.2 was much lower than that of MYB10.1, and no significant difference was found in differently bagged peaches. In contrast, the transcript level of MYB10.1 was relatively high and consistent with the anthocyanin level in the skins of peaches, indicating that MYB10.1, rather than MYB10.2, is the major and direct regulator of the peach response to bagging treatment. Furthermore, the expression pattern of PpMYB10.1 in the skin among these bagged peaches showed a high positive correlation with the light transmittance of the bags, indicating that light has a crucial role in the process of regulation; this was also observed during other peach studies (Rahim et al., 2019; Tuan et al., 2015; Zhao et al., 2019).

\section{Conclusions}

In summary, bagging treatments resulted in reduced skin redness in the peach cultivar studied. Generally, the lower the light transmittance of the fruit bag, the less redness of the skin; however, when the light intensity in the fruit bag was below a certain threshold, the change in light no longer had an impact. Anthocyanin accumulation in pericarps significantly decreased through bagging treatment compared with that of the control. This was caused by the decreased expression of several structural genes, such as $F 3 H, C H S, D F R$, and 
A

UFGT
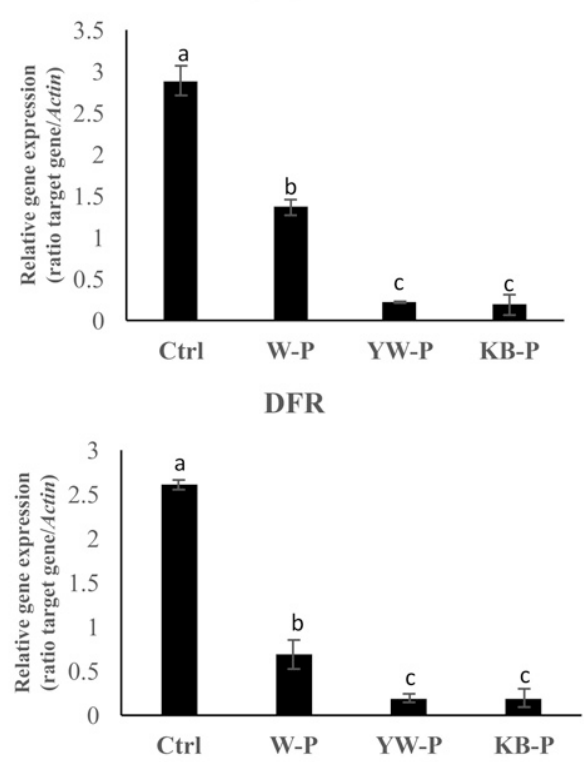

B

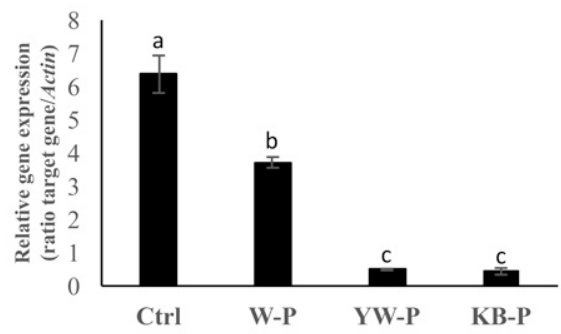

F3H
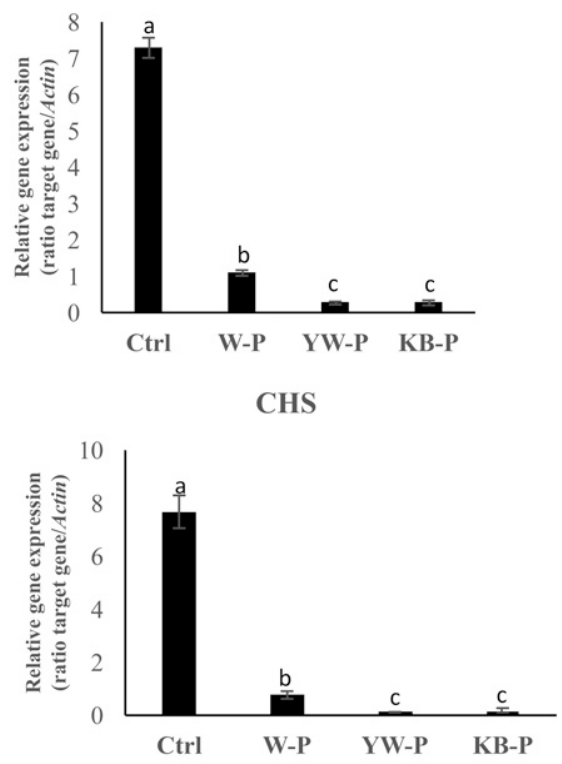

MYB10.2

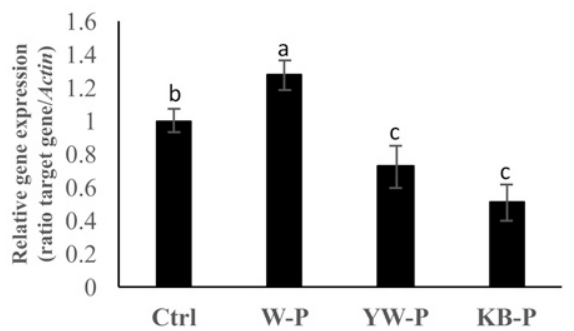

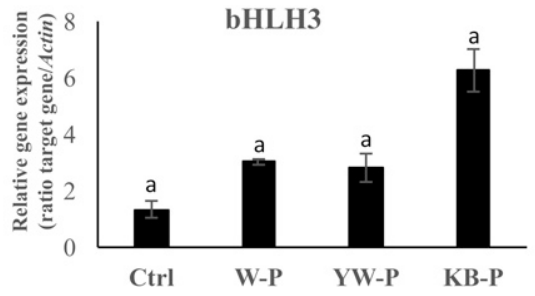

Fig. 4. (A) Effects of fruit bagging with different paper bags on the expression of related anthocyanin biosynthetic genes in peach pericarps, including UDP-glucose:flavonoid 3-O-glucosyltransferase encoding gene (UFGT), chalcone synthase encoding gene (CHS), flavonone-3-hydroxylase encoding gene $(F 3 H)$, and dihydroflavonol-4-reductase encoding gene $(D F R)$. The peach Actin gene was used as the internal control gene. (B) Effects of fruit bagging with different paper bags on the expression of related anthocyanin regulatory genes in peach pericarps, including genes encoding R2R3-MYB domains (MYB10.1 and MYB10.2) and basic helix-loop-helix domains (bHLH3). The peach Actin gene was used as the internal control gene. Values are means \pm SD of three independent biological replicates. Bars represent SE. Letters represent the nonsignificant ranges according to the least significant difference (LSD) test $(P \leq 0.01)$.

UFGT. The expression of MYB10.1 was also suppressed by fruit bagging and highly paralleled the changes in anthocyanin content, which might suggest that MYB10.1 has a major role in the light-mediated regulation of anthocyanin biosynthesis in bagged peach pericarps. Anthocyanin accumulation is a complicated process that has various responses to different environmental factors. These findings further enrich our theoretical knowledge of the regulation of anthocyanin synthesis in peach pericarps.

\section{Literature Cited}

Allan, A.C., R.P. Hellens, and W.A. Laing. 2008. MYB transcription factors that colour our fruit. Trends Plant Sci. 13:99-102.

Bai, S.L., R.Y. Tao, Y.X. Tang, L. Yin, Y.J. Ma, J.B. Ni, X.H. Yan, Q.S. Yang, Z.Y. Wu, Y.L. Zeng, and Y.W. Teng. 2019. BBX16, a B-box protein, positively regulates light-induced anthocyanin accumulation by activating MYB10 in red pear. Plant Biotechnol. J. 17:1985-1997.

Cao, K., T.Y. Ding, D.M. Mao, G.R. Zhu, W.C. Fang, C.W. Chen, X.W. Wang, and L.R. Wang. 2018. Transcriptome analysis reveals 
novel genes involved in anthocyanin biosynthesis in the flesh of peach. Plant Physiol. Biochem. 123:94-102.

Chen, W.F., M.X. Zhang, G.J. Zhang, P.M. Li, and F.W. Ma. 2019. Differential regulation of anthocyanin synthesis in apple peel under different sunlight intensities. Intl. J. Mol. Sci. 20:6060-6072.

Cheng, J., G.C. Wei, H. Zhou, C. Gu, S. Vimolmangkang, L. Liao, and Y.P. Han. 2014. Unraveling the mechanism underlying the glycosylation and methylation of anthocyanins in peach. Plant Physiol. 166:1044-1058.

Dixon, R.A. and N.L. Paiva. 1995. Stress-induced phenylpropanoid metabolism. Plant Cell 7:1085-1097.

Fan, J.L., W.X. Zhu, H.B. Kang, H.L. Ma, and G.J. Tao. 2012. Flavonoid constituents and antioxidant capacity in flowers of different Zhongyuan tree penoy cultivars. J. Funct. Foods 4:147-157.

Feng, F.J., M.J. Li, F.W. Ma, and L.L. Cheng. 2014. The effects of bagging and debagging on external fruit quality, metabolites, and the expression of anthocyanin biosynthetic genes in 'Jonagold' apple (Malus domestica Borkh.). Scientia Hort. 165:123-131.

Glover, B.J. and C. Martin. 2012. Anthocyanins. Curr. Biol. 22: 147-150.

Gonzalez, A., M. Zhao, J.M. Leavitt, and A.M. Lloyd. 2008. Regulation of the anthocyanin biosynthetic pathway by the TTG1/bHLH/ Myb transcriptional complex in Arabidopsis seedlings. Plant J. 53: 814-827.

Guo, X., Y.T. Wang, Z.F. Zhai, T.J. Huang, D. Zhao, X. Peng, C. Feng, Y.H. Xiao, and T.H. Li. 2018. Transcriptomic analysis of light-dependent anthocyanin accumulation in bicolored cherry fruit. Plant Physiol. Biochem. 130:663-677.

Han, J.L., P. Fang, X.M. Xu, X.J. Li-Zheng, H.T. Shen, and Y.P. Ren. 2015. Study of the pesticides distribution in peel, pulp and paper bag and the safety of pear bagging. Food Control 54:338-346.

He, J. and M.M. Giusti. 2010. Anthocyanins: Natural colorants with health-promoting properties. Annu. Rev. Food Sci. Technol. $1: 163-187$.

Holton, T.A. and E.C. Cornish. 1995. Genetics and biochemistry of anthocyanin biosynthesis. Plant Cell 7:1071-1083.

Hsia, C.L., B.S. Luh, and C. Co. 1965. Anthocyanin in freestone peaches. J. Food Sci. 30:5-9.

Huang, C.H., B. Yu, Y.W. Teng, J. Su, Q. Shu, Z.Q. Cheng, and L.Q. Zeng. 2009. Effects of fruit bagging on coloring and related physiology, and qualities of red chinese sand pears during fruit maturation. Scientia Hort. 121:149-158.

Jaakola, L. 2013. New insights into the regulation of anthocyanin biosynthesis in fruit. Trends Plant Sci. 18:477-483.

Jia, H.J., A. Araki, and G. Okamoto. 2005. Influence of fruit bagging on aroma volatiles and skin coloration of 'Hakuho' peach (Prunus persica Batsch). Postharvest Biol. Technol. 35:61-68.

Jimenez-Garcia, S.N., R.G. Guevara-Gonzalez, R. Miranda-Lopez, A.A. Feregrino-Perez, I. Torres-Pacheco, and M.A. Vazquez-Cruz. 2013. Functional properties and quality characteristics of bioactive compounds in berries: Biochemistry, biotechnology, and genomics. Food Res. Intl. 54:1195-1207.

Kobayashi, S., M. Ishimaru, C.K. Ding, H. Yakushiji, and N. Goto. 2001. Comparison of UDP-glucose: Flavonoid 3-O-glucosyltransferase (UFGT) gene sequences between white grapes (Vitis vinifera) and their sports with red skin. Plant Sci. 160:543-550.

Lai, B., X.J. Li, B. Hu, Y.H. Qin, X.M. Huang, H.C. Wang, and G.B. Hu. 2014. LcMYB1 Is a key determinant of differential anthocyanin accumulation among genotypes, tissues, developmental phases and ABA and light stimuli in Litchi chinensis. PLoS One 9:e86293.

Laitinen, R.A.E., M. Ainasoja, S.K. Broholm, T.H. Teeri, and P. Elomaa. 2008. Identification of target genes for a MYB-type anthocyanin regulator in Gerbera hybrida. J. Expt. Bot. 59:36913703 .

Li, C., J. Wu, K.D. Hu, S.W. Wei, H.Y. Sun, L.Y. Hu, Z. Han, G.F. Yao, and H. Zhang. 2020. PyWRKY26 and PybHLH3 cotargeted the PyMYB114 promoter to regulate anthocyanin biosynthesis and transport in red-skinned pears. Hort. Res. 7:37-48.

Li, Y.Y., K. Mao, C. Zhao, X.Y. Zhao, H.L. Zhang, H.R. Shu, and Y.J. Hao. 2012. MdCOP1 ubiquitin E3 ligases interact with MdMYB1 to regulate light-induced anthocyanin biosynthesis and red fruit coloration in apple. Plant Physiol. 160:1011-1022.

Liu, H.N., J. Su, Y.F. Zhu, G.F. Yao, A.C. Allan, C.A. Dwamena, Q. Shu, K.L. Wang, S.L. Zhang, and J. Wu. 2019a. The involvement of PybZIPa in light-induced anthocyanin accumulation via the activation of PyUFGT through binding to tandem G-boxes in its promoter. Hortic. Res. 6:134-146.

Liu, T., S. Song, Y.B. Yuan, D.J. Wu, M.J. Chen, Q.N. Sun, B. Zhang, C.J. Xu, and K.S. Chen. 2015. Improved peach peel color development by fruit bagging. Enhanced expression of anthocyanin biosynthetic and regulatory genes using white non-woven polypropylene as replacement for yellow paper. Scientia Hort. 184:142-148.

Liu, X., M. Chen, B.B. Wen, X.L. Fu, D.M. Li, X.D. Chen, D.S. Gao, L. Li, and W. Xiao. 2019b. Transcriptome analysis of peach (Prunus persica) fruit skin and differential expression of related pigment genes. Scientia Hort. 250:271-277.

Liu, Y.F., B. Zhou, Y.W. Qi, C.H. Liu, Z.D. Liu, and X.L. Ren. 2018. Biochemical and functional characterization of AcUFGT3a, a galactosyltransferase involved in anthocyanin biosynthesis in the redfleshed kiwifruit (Actinidia chinensis). Physiol. Plant. 162:409-426.

Livak, K.J. and T.D. Schmittgen. 2001. Analysis of relative gene expression data using real-time quantitative PCR and the 2(T)(-Delta Delta C) method. Methods 25:402-408.

Lo Piero, A.R., I. Puglisi, P. Rapisarda, and G. Petrone. 2005. Anthocyanins accumulation and related gene expression in red orange fruit induced by low temperature storage. J. Agr. Food Chem. 53:9083-9088.

Ma, Y.T., X.J. Zhao, H.W. Ren, H.X. Wu, M.X. Guo, Y.Z. Zhang, Z.J. He, J.M. Han, and R.J. Tong. 2018. Significant reduction of the expression of peach (Prunus persica L. Batsch) allergen-encoding genes by fruit bagging with opaque paper. J. Agr. Food Chem. 66:4051-4061.

Montefiori, M., R.V. Espley, D. Stevenson, J. Cooney, P.M. Datson, A. Saiz, R.G. Atkinson, R.P. Hellens, and A.C. Allan. 2011. Identification and characterisation of F3GT1 and F3GGT1, two glycosyltransferases responsible for anthocyanin biosynthesis in red-fleshed kiwifruit (Actinidia chinensis). Plant J. 65:106-118.

Peng, Y.Y., L.W. Kui, J.M. Cooney, T.C. Wang, R.V. Espley, and A.C. Allan. 2019. Differential regulation of the anthocyanin profile in purple kiwifruit (Actinidia species). Hort. Res. 6:3-18.

Petroni, K. and C. Tonelli. 2011. Recent advances on the regulation of anthocyanin synthesis in reproductive organs. Plant Sci. 181:219-229.

Rahim, M.A., N. Busatto, and L. Trainotti. 2014. Regulation of anthocyanin biosynthesis in peach fruit. Planta 240:913-929.

Rahim, M.A., F. Resentini, F. Dalla Vecchia, and L. Trainotti. 2019. Effects on plant growth and peproduction of a peach R2R3-MYB transcription factor overexpressed in tobacco. Front. Plant Sci. 10: 1143-1159.

Ravaglia, D., R.V. Espley, R.A. Henry-Kirk, C. Andreotti, V. Ziosi, R.P. Hellens, G. Costa, and A.C. Allan. 2013. Transcriptional regulation of flavonoid biosynthesis in nectarine (Prunus persica) by a set of R2R3 MYB transcription factors. BMC Plant Biol. 13:68-81.

Reig, G., I. Iglesias, F. Gatius, and S. Alegre. 2013. Antioxidant capacity, quality, and anthocyanin and nutrient contents of several peach cultivars [Prunus persica (L.) Batsch] grown in Spain. J. Agr. Food Chem. 61:6344-6357.

Sharma, R.R., S.V.R. Reddy, and M.J. Jhalegar. 2014. Pre-harvest fruit bagging: A useful approach for plant protection and improved post-harvest fruit quality - A review. J. Hort. Sci. Biotechnol. 89: 101-113.

Sun, C.D., Y.X. Zheng, Q.J. Chen, X.L. Tang, M. Jiang, J.K. Zhang, X. Li, and K.S. Chen. 2012. Purification and anti-tumour activity of cyanidin-3-O-glucoside from chinese bayberry fruit. Food Chem. 131:1287-1294. 
Takos, A.M., F.W. Jaffe, S.R. Jacob, J. Bogs, S.P. Robinson, and A.R. Walker. 2006. Light-induced expression of a MYB gene regulates anthocyanin biosynthesis in red apples. Plant Physiol. 142:1216-1232.

Tanaka, Y., N. Sasaki, and A. Ohmiya. 2008. Biosynthesis of plant pigments: Anthocyanins, betalains and carotenoids. Plant J. 54: 733-749.

Tsuda, T., M. Yamaguchi, C. Honda, and T. Moriguchi. 2004. Expression of anthocyanin biosynthesis genes in the skin of peach and nectarine fruit. J. Amer. Soc. Hort. Sci. 129:857-862.

Tuan, P.A., S.L. Bai, H. Yaegaki, T. Tamura, S. Hihara, T. Moriguchi, and K. Oda. 2015. The crucial role of PpMYB10.1 in anthocyanin accumulation in peach and relationships between its allelic type and skin color phenotype. BMC Plant Biol. 15:1-14.

Winkel-Shirley, B. 2001. Flavonoid biosynthesis. A colorful model for genetics, biochemistry, cell biology, and biotechnology. Plant Physiol. 126:485-493.

Xi, W.P., J. Feng, Y. Liu, S.K. Zhang, and G.H. Zhao. 2019. The R2R3-MYB transcription factor PaMYB10 is involved in anthocyanin biosynthesis in apricots and determines red blushed skin. BMC Plant Biol. 19:287-300.

Ye, J.B., G.Y. Wang, J.P. Tan, J.R. Zheng, X. Zhang, F. Xu, S.Y. Cheng, Z.X. Chen, W.W. Zhang, and Y.L. Liao. 2019. Identification ofcandidate genes involved in anthocyanin accumulation using Illmuinabased RNA-seq in peach skin. Scientia Hort. 250:184-198.

Zhang, H.N., W.C. Li, H.C. Wang, S.Y. Shi, B. Shu, L.Q. Liu, Y.Z. Wei, and J.H. Xie. 2016. Transcriptome profiling of light-regulated anthocyanin biosynthesis in the pericarp of litchi. Front. Plant Sci. 7:963-977.

Zhao, Y., W.Q. Dong, Y.C. Zhu, A.C. Allan, L.W. Kui, and C.J. Xu. 2019. PpGST1, an anthocyanin-related glutathione S-transferase gene, is essential for fruit coloration in peach. Plant Biotechnol. J. 18:1284-1295.

Zheng, H.X., S.S. Qi, J. He, C.Y. Hu, H. Han, H. Jiang, and X.S. Li. 2020. Cyanidin-3-glucoside from black rice ameliorates diabetic nephropathy via reducing blood glucose, suppressing oxidative stress and inflammation, and regulating transforming growth factor beta1/ smad expression. J. Agr. Food Chem. 68:4399-4410.

Zhou, Y., D. Guo, J. Li, J. Cheng, H. Zhou, C. Gu, S. Gardiner, and Y.P. Han. 2013. Coordinated regulation of anthocyanin biosynthesis through photorespiration and temperature in peach (Prunus persica $\mathrm{f}$. atropurpurea). Tree Genet. Genomes 9:265-278.

Zoratti, L., K. Karppinen, A.L. Escobar, H. Haggman, and L. Jaakola. 2014. Light-controlled flavonoid biosynthesis in fruit. Front. Plant Sci. 5:534-549. 
Supplemental Table 1. Primers used for quantitative real-time PCR analysis of anthocyanin biosynthetic and regulatory genes in peaches.

\begin{tabular}{|c|c|c|c|c|c|}
\hline $\mathrm{Gene}^{\mathrm{z}}$ & Gene symbol & Phytozome no. & Primer & Primer sequence $\left[5^{\prime}-3^{\prime}\right]$ & Amplicon (bp) \\
\hline \multirow[t]{2}{*}{ UFGT } & LOC18785045 & $\mathrm{ppa} 005162 \mathrm{~m}$ & Forward & ACGACGTTACTGGTTGCCTA & 106 \\
\hline & & & Reverse & ATAAGCTCCTTCTCCGGTGG & \\
\hline \multirow[t]{2}{*}{ DFR } & LOC18788884 & ppa008069m & Forward & CTCAGCAGGAACCGTGAATG & 116 \\
\hline & & & Reverse & AGGCGAAGTACATCCAACCA & \\
\hline \multirow[t]{2}{*}{$\mathrm{CHS}$} & LOC18793306 & ppa006888m & Forward & AGATGGACTGGATTGGGGTG & 124 \\
\hline & & & Reverse & TGCAGCTTCCTCTTCCTTCA & \\
\hline \multirow[t]{2}{*}{ F3H } & LOC18770366 & ppa007636m & Forward & AGGGAGAAGCTGTGCAAGAT & 130 \\
\hline & & & Reverse & TCGTCGCTGTACTTCTGTGT & \\
\hline \multirow[t]{2}{*}{ MYB10.1 } & LOC18783018 & ppa026640m & Forward & TGGGTGTGAGAAAAGGAGCT & 125 \\
\hline & & & Reverse & СТTCCTGCACCTGTTCAACC & \\
\hline \multirow[t]{2}{*}{ MYB10.2 } & LOC18783815 & ppa016711m & Forward & GGTTGAACAGGTGCAGGAAG & 149 \\
\hline & & & Reverse & TCGAGCAATCAATGACCACC & \\
\hline \multirow[t]{2}{*}{ bHLH3 } & LOC18767836 & ppa002884m & Forward & GGCATTTCTTGGGCAACTGA & 131 \\
\hline & & & Reverse & AAGGTGGAGGAGGGAGAGAT & \\
\hline \multirow[t]{2}{*}{ Actin } & LOC18773538 & ppa007214m & Forward & AGCAACTGGGATGACATGGA & 103 \\
\hline & & & Reverse & TGAGAGGTGCTTCAGTGAGG & \\
\hline
\end{tabular}

$\overline{\mathrm{z}} b H L H=$ basic helix-loop-helix, $C H S=$ chalcone synthase, $D F R=$ dihydroflavonol 4-reductase, $F 3 H=$ flavanone 3-hydroxylase, $U F G T=$ UDP-glucose:flavonoid-3-O-glucosyltransferase. 